\title{
DYNAMIC TESTING SYSTEM FOR ROCKS UNDER IN SITU STRESSES
}

\author{
Wei Yao, Kaiwen Xia \\ Department of Civil Engineering \\ University of Toronto \\ Toronto, Canada \\ wei.yao@mail.utoronto.ca, kaiwen.xia@utoronto.ca
}

\begin{abstract}
Rocks may be subjected to dynamic disturbances while under high in situ stresses. When disturbed by dynamic loads from blasting, seismicity or rockbursts, the underground structures would be vulnerable to failure. Depending on the distance from the underground opening, the in situ stress states change from hydrostatic in the far-field, to triaxial in the intermediate distance, and to the pre-tension nearby the opening. Thus, SHPB testing system is further adjusted with confining pressure system into dynamic testing system of rocks under different in situ states. In the experiment with this dynamic testing system, the Brazilian disc rock specimens are first subjected to pre-stresses simulating in-situ stresses underground (including pre-tension, hydrostatic confinement, and triaxial confinement) and then loaded dynamically using the modified SHPB system. The dependence of dynamic tensile strength of the rock material on the static pre-stress and loading rate is investigated. These experimental results will be of great importance in the design and safety of underground rock engineering projects.
\end{abstract}

Keywords-SHPB; Pre-stress; Hydrostatic Confinement; Triaxial Stress; Rate dependence

\section{INTRODUCTION}

With the development of underground space, it is necessary to study the mechanical properties of underground rocks, which are subjected to high lithostatic and tectonic stress. Problems involving underground rocks are common in many important economic fields including hydropower, transportation, mining and defense infrastructure. Deep rock structures are prone to dynamic loads such as blast mining, rock burst and small seismic events, so it is necessary to simulate the underground stress state and conduct designed dynamic experiments to investigate the dynamic properties of underground rocks. Depending on the distance from the underground opening, the in situ stress states change from hydrostatic in the far-field, to triaxial in the intermediate distance, and to the pre-tension nearby the opening, as shown in Fig. 1.

Dynamic mechanical properties of engineering materials have been investigated for many years and various devices are used to conduct experiments on these materials. One of the most frequently used techniques is the split Hopkinson pressure bar (SHPB) system. Using the SHPB technique, dynamic mechanical properties such as compressive strength, tensile strength and fracture toughness have been studied on engineering materials, mostly under uniaxial loading conditions [1]. However, underground rocks are subjected to in situ stresses and occasional dynamic loadings. Most research on axial compression properties of rock-like materials under the preloading situations are conducted on ceramics and concretes under lateral confinement, either passive or active [2]. Gong and Malvern [3] proposed a way to provide passive confinement on concrete specimens in the SHPB system. Chen and Ravichandran [4] adopted the passive confinement method to investigate the dynamic properties of ceramics. Song and Chen et al. [5] studied confinement effects on the dynamic compressive properties of an epoxy syntactic foam, considering the effect of both the loading rate and passive lateral confinement. Christen and Swanson et al. [6] designed dynamic mechanical experiments under active confinement. Gary and Bailly [7] adopted the technique and found the confining pressure more stable with air as the medium. They conducted experiments on concrete and proposed a mesomechanical model. Lindholm and Yeakley et al. [8] proposed the experimental method under hydrostatic confining pressure in 1974. $\mathrm{Li}$ and Zhou et al. [9] and $\mathrm{Zuo}$ and $\mathrm{Li}$ et al. [10] investigated the dynamic response of rocks under both axial and active lateral confinement. Frew and Akers et al. [11] improved Lindholm's device and developed a hydrostatic apparatus based on SHPB system.

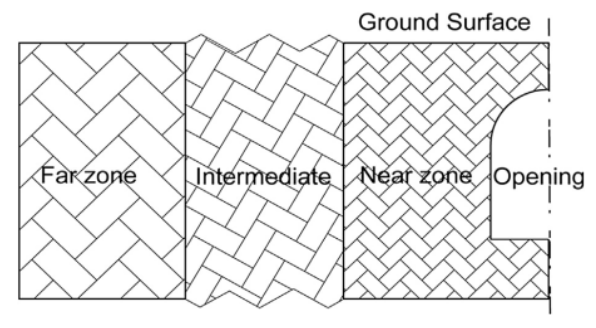

Figure 1. Zoning of the confining stress states around an underground opening

It is worth noting that, in previous research, there are not many results of tensile experiments on pre-stressed rocks, and 
if so, all of them are under quasi-static stress states. Some researchers conducted Brazilian disc (BD), shear and torsion tests on rocks under hydrostatic pressure [12, 13] and they found that the strength of rock increases with the hydrostatic pressure. Vasarhelyi [14] investigated the influence of confinement on the mode I fracture of Gneiss. Al-Shayea and Khan et al. [15] tested straight notched Brazilian disk (SNBD) specimens under diametrical compression to study the influence of confinement on fracture toughness of limestone. Chen and Zhang [16] studied the influence of confinement on rock fracture toughness using notch-hole combined Brazilian disc specimens and Funatsu and Seto et al. [17] did the same tests on notched semi-circular bend (NSCB) specimens and they found the same increasing trend with confining pressure.

Therefore, there have been many improvements in dynamic testing of rocks with the static stress states; however, the research and testing system concerning various dynamic response (e.g. compression and tension strength, fracture toughness) of rocks subjected to different pre-stresses (i.e. pretension, hydrostatic confinement, and triaxial confinement) are still deficient. In this paper, a SHPB testing system is adjusted with confining pressure system for dynamic response of rocks under different pre-stress states. To calibrate this testing system, the Brazilian disc rock specimens are first subjected to simulating in-situ stresses underground (including pre-tension, hydrostatic confinement, and triaxial confinement) and then loaded dynamically using the modified SHPB system. The dependence of dynamic tensile strength of the rock material on the static pre-stress and loading rate is investigated. The calibrated testing system will be importantly applied to the design and safety of underground rock engineering projects.

\section{SHPB SYSTEM FOR ROCKS UNDER PRE- TENSION}

\section{A. The Pre-tension SHPB System}

The modified SHPB system for pre-tension test includes three bars (a striker bar, an incident bar, and a transmitted bar) [18] and the pre-tension system (Fig. 2). The elastic bars are made of high strength maraging steel. The pre-tension system is mainly composed of a pressure chamber that provides axial preload to the bars and specimen, and a rigid mass at the incident bar end that is connected to the chamber by tie-rods. The pre-tension system is similar to that innovated by Frew et al. [11], who developed a modified SHPB system for dynamic tests under hydrostatic confinement. However, there is a main difference between the current design and those of Few et al. [11] and Zhou et al. [19]. In their designs, the bars are connected by the tie-rods from the impact end of the incident bar to the free end of the transmitted bar, while in the current design, the bars are connected near the other end of the incident bar through a flange (Fig. 2). The total length of the compressed bars in the current design is much shorter and thus is less prone to buckling.

The recording system consists of the foil strain gauges, a signal conditioner, and an oscilloscope. There are two strains gauges on each bar attached at the symmetrical position, and they are connected to a signal conditioner through a Wheatstone bridge. The oscilloscope is connected to the signal conditioner using two channels, one for the signal on the incident bar and the other for the signal on the transmitted bar.

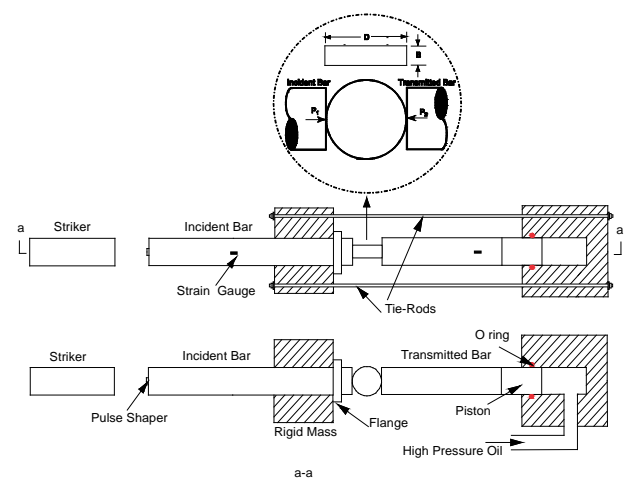

Figure 2. Schematics of the pre-tension SHPB system

\section{B. The Validity of Dynamic Test with Pre-tension}

During the tests, the static pre-tension is applied to the specimen by the pressure loading unit attached to the end of the transmitted bar through the elastic bars and flange supported by a rigid mass. When the desired pre-tension is achieved, dynamic loading is applied from the impact of the striker bar on the free end of the incident bar. The incident pulse propagates along the incident bar before it hits the specimen, leading to a reflected stress wave and a transmitted stress wave that are recorded by the strain gauges attached on the incident and transmitted bar surfaces. The motion induced by the incident wave is to the right and thus the flange has no effect on the wave propagation. The strains of incident wave, reflected wave and transmitted wave are denoted by $\varepsilon_{i}, \varepsilon_{r}$ and $\varepsilon_{t}$, respectively.

Based on the one dimensional stress wave theory, and assuming stress equilibrium during loading [18] (i.e., $\varepsilon_{i}+\varepsilon_{r}=\varepsilon_{t}$ ), the history of the force on the specimen is:

$$
P(t)=P_{0}+P_{d}(t)
$$

where $P_{0}$ is the static preload on the bars, $P_{d}(t)$ is the dynamic force history on the bars after the impact. The tensile stress history at the center of the disc specimen can be determined as:

$$
\sigma(t)=\sigma_{0}+\sigma_{d}(t)=\frac{A_{0} E_{0} \varepsilon_{t}(t)}{\pi R B}
$$

where $\sigma_{0}$ is the pre-tension at the center of the disc, and

$$
\sigma_{0}=\frac{P_{0}}{\pi R B}
$$

where $\sigma_{d}(t)$ is the dynamic tensile stress, $E_{0}$ is the Young's Modulus of the bars, $A_{0}$ is the cross-sectional area of the bars; $R$ is the radius of the specimen and $B$ is the thickness of the specimen. The tensile strength is the maximum value of the tensile stress when the rock specimen is damaged. There is an 
approximately linear region in $\sigma_{d}(t)$ (Fig. 3), and its slope is taken as the loading rate.

Similar to the traditional dynamic rock tension experiments in the SHPB system, the validity of dynamic test with pretension is achieved by the force balance in the specimen during the experiments. This can be researched by using pulse shaper technique [18]. Fig. 3 shows the force history according to the strain gauges attached on the incident bar and the transmitted bar. The force on one side of the specimen is the sum of forces by the incident and reflected stress waves (marked as In.+Re), and the force on the other side of the specimen is by the transmitted stress wave (marked as Tr). The initial force on the transmitted bar corresponds to the preload on the specimen, which is $P_{0}$ as in Eq. (1). It can be seen from Fig. 3 that the dynamic forces on both sides of the specimens are almost identical during $80 \mu \mathrm{s}$ to $160 \mu \mathrm{s}$, which is the main dynamic loading period. It is also noted that the forces on the two ends of the specimen are different before $80 \mu \mathrm{s}$. The reason is that before the dynamic impact, the specimen is subject to the static preload through the transmitted bar, which is already compressed and thus the strain gauge on the transmitted bar measured the preload. However, the incident bar is free before impact loading and thus there is no deformation measured by the strain gauge on the incident bar at the beginning. The force difference at time zero in Fig. 3 is the static preload applied on the rock specimen, which is $10.07 \mathrm{KN}$ in this case, corresponding to the pre-tension of $10 \mathrm{MPa}$ at the specimen center.

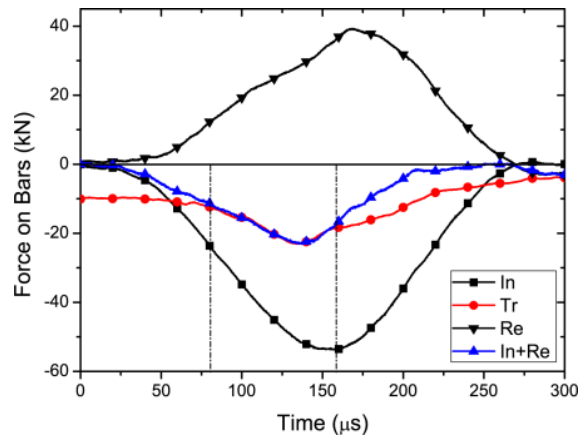

Figure 3. Dynamic force balance in a typical pre-tension SHPB test

\section{Dynamic Brazilian Test with Pre-tension}

Five groups of BD rock specimens (with static tensile strength of $12.8 \mathrm{MPa}$ ) under the pre-tension of $0 \mathrm{MPa}, 2 \mathrm{MPa}$, $4 \mathrm{MPa}, 8 \mathrm{MPa}$, and $10 \mathrm{MPa}$ are tested under different loading rates.

During each test, the tensile strength is the maximum value of the tensile stress history. Fig. 4 illustrates the dynamic tensile strength versus loading rate. It is obvious that the dynamic strength increases with the loading rate, revealing the phenomenon of rate dependency that is common for engineering materials, such as rock [20], concrete [21, 22], ceramic $[23,24]$.

Apart from the rate dependency mentioned above, what can be seen from Fig. 4 is that the dynamic tensile strength of the rock decreases with the increase of the pre-tension when subjected to the same loading rate. For example, when the loading rate is approximately $190 \mathrm{GPa} / \mathrm{s}$, the dynamic tensile strength is $19.8 \mathrm{MPa}$ when the specimen is loaded stress-free being subjected to dynamic loading. However, the dynamic tensile strength of the one with $10 \mathrm{MPa}$ pre-tension is 11.7 $\mathrm{MPa}$, which is 8.1 MPa lower than the former. The decrease of dynamic tensile strength is caused by the opening of microcracks when the specimen bears the pre-tension stress, which is consistent to the results reported by Xia et al. that the microstructures affect the dynamic stress of rock specimens [2].

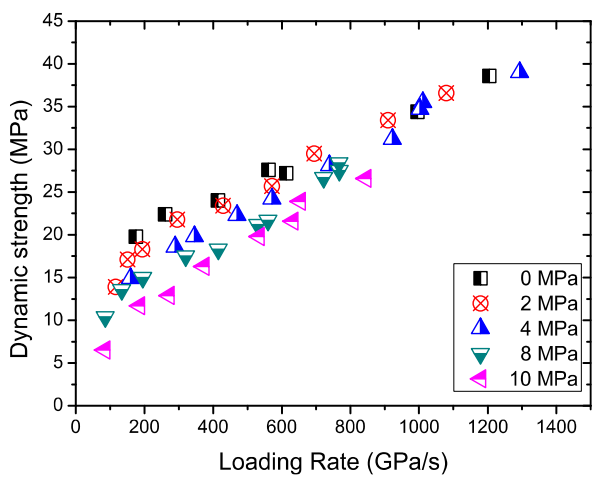

Figure 4. The dynamic strength versus loading rate for different pre-tensions

\section{SHPB SYSTEM FOR ROCKS UNDER HYDROSTATIC STRESS}

\section{A. The SHPB System with Hydrostatic Confinement}

The modified SHPB apparatus for tri-axial stress state includes the three bars (a striker bar, an incident bar, and a transmitted bar) [18] and a hydraulic system, as shown in Fig. 5. The elastic bars are made of the same material as that for the pre-tension test. The hydraulic system is mainly composed of a cylinder that applies lateral confinement to the rock specimens (Cylinder 1), and a pressure chamber that provides axial preload (Cylinder 2) to the bars and specimen. The only difference between the apparatus in Fig. 2 and the apparatus in Fig. 5 is Cylinder 1 that provides the lateral confining pressure. The recording system is same as the pre-tension SHPB system (Fig. 5).

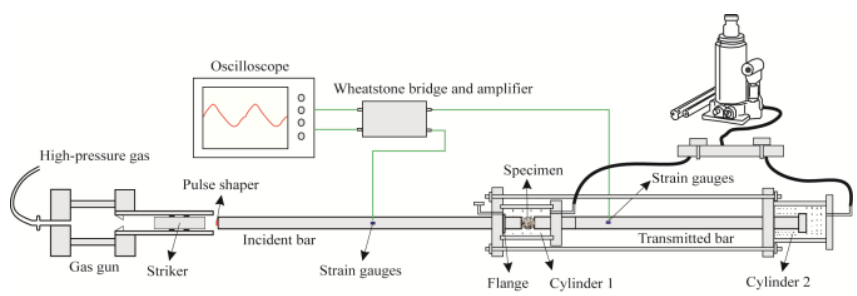

Figure 5. Schematics of the modified SHPB system for Tri-axial test

As shown in Fig. 6, the two cylinders are connected to the same hydraulic press by separate valves. When the two valves are open at the same time, the rock specimen would be in hydrostatic stress state, regardless of the shape of the rock specimen. Denote $A_{b}$ as the cross area of the bars, $A_{s}$ as the 
contact area between the specimen and the transmitted bar; denote $\sigma_{0}$ as the oil pressure provided by the pump, $\sigma_{1}$ and $\sigma_{2}$ as the stresses of the specimen at the transmitted bar end and the incident bar end, respectively.

When the two valves are open at the same time, both the lateral confining stress on the specimen and the stress on the transmitted bar are equal to the oil pressure $\sigma_{0}$. Since the transmitted bar is force balanced, the force on the transmitted bar at the specimen end equals that at the pump end. The force at the specimen end is composed of two parts, the one by the pressure oil $\sigma_{0}\left(A_{b}-A_{s}\right)$ and the one by the specimen $\sigma_{1} A_{s}$; the force at the pump end is $\sigma_{0} A_{b}$. So, the force balance of the transmitted bar leads to:

$$
\sigma_{0}\left(A_{b}-A_{\mathrm{s}}\right)+\sigma_{1} A_{\mathrm{s}}=\sigma_{0} A_{b}
$$

solve Eq. (4) we can get

$$
\sigma_{1}=\sigma_{0}
$$

similarly, we can get

$$
\sigma_{0}=\sigma_{1}=\sigma_{2}
$$

Hence the specimen is in hydrostatic stress state when the two valves are both open at the same time. It can be shown in the similar way that for a specimen with arbitrary shape, as long as it is symmetric with respect to the loading axis, the specimen is under hydrostatic confinement if the pressure in both cylinders is identical.

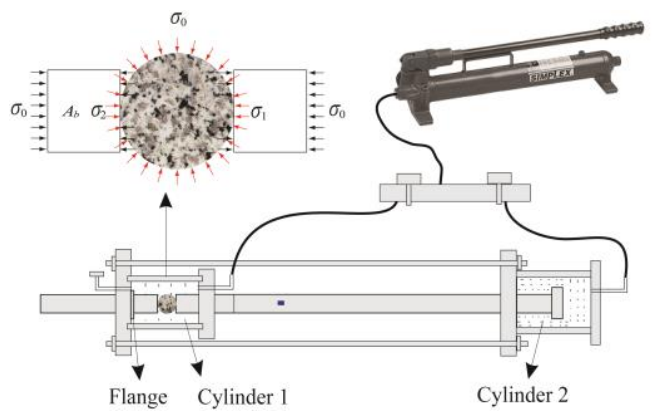

Figure 6. Hydrostatic stress of rock materials in a SHPB test

\section{B. The Validity of Dynamic Test with Hydrostatic Confinement}

Similar to the dynamic test with pre-tension in SHPB system, the dynamic test with hydrostatic confinement in SHPB system is valid when the force balance in the specimen during the experiments is achieved. This is also facilitated by using pulse shaper technique. In addition, enough loads are required to break the confined specimen so the striker is launched with sufficient air pressure every time with different pulse shapers. Different dimensions of the shapers made of different materials lead to different loading rates on the rock specimens.

Fig. 7 shows a typical force history of the two loading ends of the specimen measured by the strain gauges. On one side of the specimen is the sum of forces by the incident and reflected stress waves, which is marked as In.+Re. in the figure, and on the other side is the force by the transmitted stress wave, marked as Tr. It can be seen that the dynamic forces on both sides of the specimens are identical during the loading and unloading period, demonstrating the validity of the test. It also shows that although the incident force is as high as about 200 $\mathrm{KN}$, the force on the specimen is only about $50 \mathrm{KN}$, which is 25 percent of the incident force, and this is why the striker is launched at high air pressure every time to make sure the load is high enough to break the confined specimens.

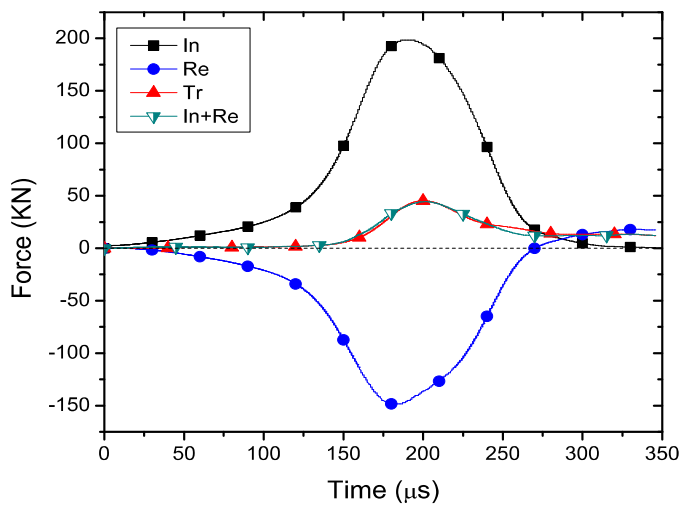

Figure 7. Dynamic force balance in a typical SHPB test with confined Brazilian disc specimen

\section{Dynamic Brazilian Test with Hydrostatic Confinement}

Five groups of specimens are tested under the hydrostatic confinements of $0 \mathrm{MPa}, 5 \mathrm{MPa}, 10 \mathrm{MPa}, 15 \mathrm{MPa}$, and $20 \mathrm{MPa}$. The tensile stress history at the center can be determined as:

$$
\sigma(t)=\frac{2 A_{b} E_{b} \varepsilon_{t}(t)}{\pi B D}
$$

where $E_{b}$ is the Young's Modulus of the bars, $A_{b}$ is the crosssectional area of the bars; $B$ and $D$ are the thickness and the diameter of the specimen, respectively. The tensile strength is the maximum value of the tensile stress when the confined rock specimen is damaged.

Fig. 8 illustrates the dynamic tensile strength versus loading rate. It is obvious that the dynamic strength increases with the loading rate.

Based on the fitted curves in Fig. 8. It is obvious that the dynamic tensile strength increases with both the hydrostatic stress and the loading rate. It can be also seen that the increment of the dynamic tensile strength decreases with the loading rate and the hydrostatic stress. This indicates that when the confining pressure reaches a certain value, or at a certain underground depth, the dynamic tensile strength of rock materials would mainly depend on the loading rate, it does not 
change much as the confinement increases further under the same loading rate.

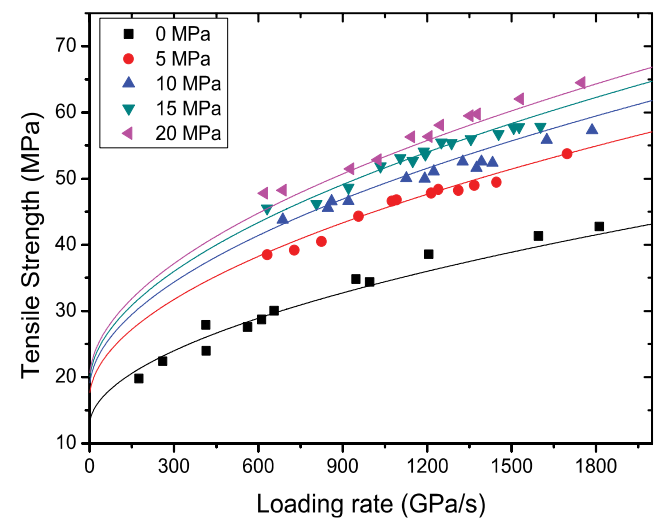

Figure 8. Dynamic tensile testing results under different loading rates and hydrostatic pre-stress, and data fitting of the results

\section{SHPB SYSTEM FOR ROCKS UNDER TRI-AXIAL STRESS STATES}

\section{A. The SHPB System for Tri-axial Stress State}

The modified SHPB apparatus for tri-axial stress state is the same as that for hydrostatic confinement test, as shown in Fig. 5. The experimental design is that we first exert a hydrostatic stress to the BD specimen through the cylinders and the press, by opening the two valves at the same time. When the hydrostatic stress is achieved, the valve controlling the lateral confinement is closed, and the cylinder at the transmitted bar end is used to provide more axial load to the specimen for pretension so that the specimen is under triaxial stress conditions (Fig. 9).

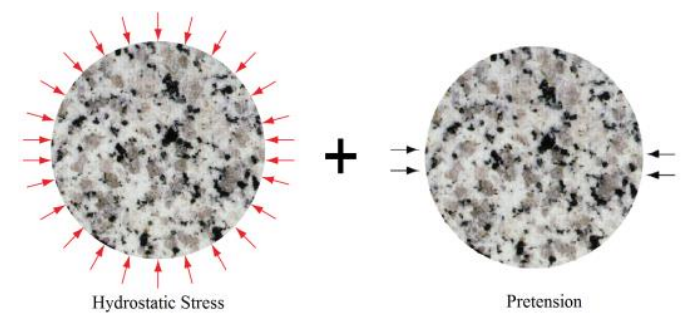

Figure 9. Experimental design with different hydrostatic stress and pretension

After the calculation of the stress on the specimen, the stress exerted on the bar is then determined to achieve the prestress conditions before the impact is launched. For example, when the hydrostatic stress is $5 \mathrm{MPa}$, then the Brazilian tensile strength of the specimen is $17.8 \mathrm{MPa}$, which is the sum of the hydrostatic stress and the $\mathrm{BD}$ strength without any confinement. Then with the design that the pretension is $20 \%$ of the strength, which is $3.56 \mathrm{MPa}$ on the specimen. Through the correlation between the tensile stress on the specimen and the stress at the bar specimen interface, the load needed on the bar can be determined.
Denote $\sigma_{t}$ as the tensile stress at the specimen center, through the correlation between the tensile stress at the specimen center and the stress at the specimen-bar interface,

$$
\Delta \sigma_{\mathrm{t}}=\frac{2 P}{\pi B D}=\frac{1}{2} \frac{\pi D_{b}^{2} \sigma_{b}}{\pi B D}=\frac{D_{b}{ }^{2} \sigma_{b}}{2 B D} .
$$

where $P$ is the force on the bar, $B$ and $D$ are the thickness and diameter of the specimen, while $\sigma_{b}$ is the stress on the bar, and $D_{b}$ is the diameter of the bars. So, the stress on the bar should be calculated from Eq. (9) to achieve the desired pre-stress conditions.

$$
\sigma_{b}=\frac{2 B D \sigma_{t}}{D_{b}^{2}}
$$

With hydrostatic confinement, the axial stress applied on transmitted bar is:

$$
\sigma_{b}=\frac{2 B D \sigma_{t}}{D_{b}^{2}}+\sigma_{0}
$$

where $\sigma_{0}$ is the hydrostatic stress on the specimen.

\section{B. Dynamic Brazilian Test under Tri-axial Stress State}

Similar to the dynamic test with hydrostatic confinement in SHPB system, the dynamic test for the tri-axial stress state is valid by using the achievement of the force balance in the specimen during the experiments. This is also facilitated by using pulse shaper technique.

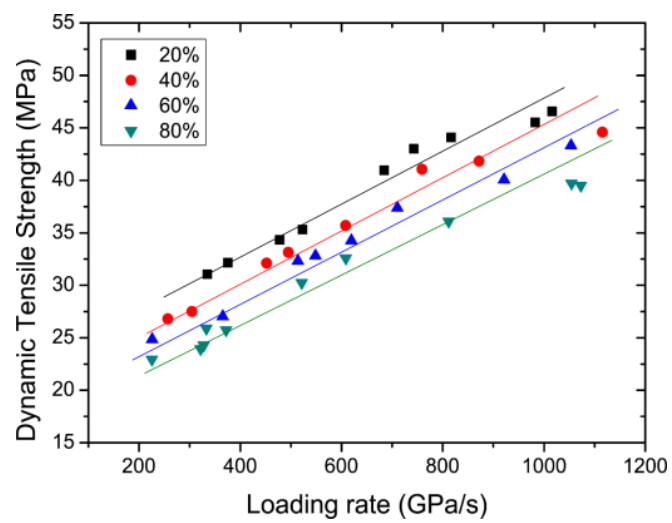

Figure 10. Total strength of rock specimen under 5MPa hydrostatic stress and pre-tension

The tensile stress history at the center can be determined as:

$$
\sigma(t)=\frac{2 A_{b} E_{b} \varepsilon_{t}(t)}{\pi B D}
$$


where $E_{b}$ is the Young's Modulus of the bars, $A_{b}$ is the crosssectional area of the bars; $B$ and $D$ are the thickness and the diameter of the specimen, respectively. The tensile strength is the maximum value of the tensile stress when the confined rock specimen is damaged.

Four groups of specimens are tested under the hydrostatic confinements of $5 \mathrm{MPa}$ and the pretensions are $20 \%, 40 \%$, $60 \%$ and $80 \%$ of the tensile strength under the corresponding hydrostatic stress state, as shown in Fig. 10. Fig. 10 illustrates the correlation between tensile strength and the loading rate when the rock specimens are under $5 \mathrm{MPa}$ hydrostatic stress and various pretensions, respectively. It can be observed that the tensile strength increases with the loading rate almost linearly, revealing the phenomenon of rate dependency that is common for engineering materials. Besides the rate dependency mentioned above, it is also obvious from Fig. 10 that the dynamic tensile strength of the rock decreases with triaxial stress states, which is also observed from the pre-tension tests in Section II.

\section{CONCLUSIONS}

When the underground rocks under high in situ stresses are disturbed by dynamic loads from blasting, seismicity or rockbursts, the underground structures would be vulnerable to failure. In situ stress states of the underground openings change from hydrostatic in the far-field, to triaxial in the intermediate distance, and to the pre-tension nearby the opening. In order to investigate the dynamic behaviors (e.g. compression and tension strength, fracture toughness) of rocks under in situ states, a dynamic testing system of rocks under different in situ states (i.e. pre-tension, hydrostatic confinement, and triaxial confinement) is modified with the SHPB testing system and confining pressure system.

To calibrate the dynamic testing system of rocks under in situ states, the Brazilian disc rock specimens are first subjected to simulating in-situ stresses underground (including pretension, hydrostatic confinement, and triaxial confinement) and then loaded dynamically through the modified SHPB system. The advantages of the design for the dynamic testing system is given and the validity of dynamic BD rock tests performed in this dynamic testing system was discussed. Based on this system, the dependence of dynamic tensile strength of the rock material on the static pre-stress and loading rate is investigated. The calibrated testing system will be importantly applied to the design and safety of underground rock engineering projects.

\section{REFERENCES}

[1] K. Xia, W. Yao. "Dynamic rock tests using split Hopkinson (Kolsky) bar system-A review," Int J Rock Mech Min, vol. 7, pp. 27-59, 2015.

[2] K. Xia, M. H. B. Nasseri, B. Mohanty, F. Lu, R. Chen, S. N. Luo. "Effects of microstructures on dynamic compression of Barre granite," Int J Rock Mech Min, vol. 45, pp. 879-887, 2008.
[3] J. C. Gong, L. E. Malvern. "Passively Confined Tests of Axial Dynamic Compressive Strength of Concrete," Exp Mech, vol. 30, pp. 55-59, 1990.

[4] W. Chen, G. Ravichandran. "An experimental technique for imposing dynamic multiaxial-compression with mechanical confinement," Exp Mech, vol. 36, pp. 155-158, 1996.

[5] B. Song, W. N. Chen, T. Yanagita, D. J. Frew. "Confinement effects on the dynamic compressive properties of an epoxy syntactic foam," Compos Struct, vol. 67, pp. 279-287, 2005.

[6] R. J. Christen, S. R. Swanson, W. S. Brown. "Split-Hopkinson-Bar Tests on Rock under Confining Pressure," Exp Mech, vol. 12, pp. 508-513, 1972.

[7] G. Gary, P. Bailly. "Behaviour of quasi-brittle material at high strain rate. Experiment and modelling," Eur J Mech a-Solid, vol. 17, pp. 403420, 1998.

[8] U. S. Lindholm, L. M. Yeakley, A. Nagy. "Dynamic Strength and Fracture Properties of Dresser Basalt," Int J Rock Mech Min, vol. 11, pp. 181-191, 1974.

[9] X. B. Li, Z. L. Zhou, T. S. Lok, L. Hong, T. B. Yin. "Innovative testing technique of rock subjected to coupled static and dynamic loads," Int J Rock Mech Min, vol. 45, pp. 739-748, 2008.

[10] Y. J. Zuo, X. B. Li, Z. L. Zhou, C. D. Ma, Y. P. Zhang, W. H. Wang. "Damage and failure rule of rock undergoing uniaxial compressive load and dynamic load," J Cent South Univ T, vol. 12, pp. 742-748, 2005.

[11] D. J. Frew, S. A. Akers, W. Chen, M. L. Green. "Development of a dynamic triaxial Kolsky bar," Meas Sci Technol, vol. 21, pp. 105704(105710), 2010.

[12] E. C. Robertson. "Experimental Study of the Strength of Rocks," Geol Soc Am Bull, vol. 66, pp. 1275-1314, 1955.

[13] J. C. Jaeger, E. R. Hoskins. "Rock Failure under Confined Brazilian Test," J Geophys Res, vol. 71, pp. 2651-2659, 1966.

[14] B. Vasarhelyi. "Influence of pressure on the crack propagation under mode I loading in anisotropic gneiss," Rock Mech Rock Eng, vol. 30, pp. 59-64, 1997.

[15] N. A. Al-Shayea, K. Khan, S. N. Abduljauwad. "Effects of confining pressure and temperature on mixed-mode (I-II) fracture toughness of a limestone rode," Int J Rock Mech Min, vol. 37, pp. 629-643, 2000.

[16] M. Chen, G. Q. Zhang. "Laboratory measurement and interpretation of the fracture toughness of formation rocks at great depth," J Petrol Sci Eng, vol. 41, pp. 221-231, 2004.

[17] T. Funatsu, M. Seto, H. Shimada, K. Matsui, M. Kuruppu. "Combined effects of increasing temperature and confining pressure on the fracture toughness of clay bearing rocks," Int J Rock Mech Min, vol. 41, pp. 927938, 2004.

[18] Y. X. Zhou, K. Xia, X. B. Li, H. B. Li, G. W. Ma, J. Zhao, et al. "Suggested methods for determining the dynamic strength parameters and mode-I fracture toughness of rock materials," Int J Rock Mech Min, vol. 49, pp. 105-112, 2012.

[19] Z. Zhou, X. Li, Y. Zou, Y. Jiang, G. Li. "Dynamic Brazilian tests of granite under coupled static and dynamic loads," Rock Mech Rock Eng, vol. 47, pp. 495-505, 2014.

[20] Q. B. Zhang, J. Zhao. "A Review of Dynamic Experimental Techniques and Mechanical Behavior of Rock Materials," Rock Mech Rock Eng, vol. 47, pp. 1411-1478, 2013.

[21] G. Cusatis. "Strain-rate effects on concrete behavior," Int J Impact Eng, vol. 38, pp. 162-170, 2011.

[22] K. Fujikake, T. Senga, N. Ueda, T. Ohno, M. Katagiri. "Effects of Strain Rate on Tensile Behavior of Reactive Powder Concrete," J Adv Concr Technol, vol. 4, pp. 79-84, 2006.

[23] N. S. Brar, Z. Rosenberg. "Brittle Failure of Ceramic Rods under Dynamic Compression," J Phys-Paris, vol. 49, pp. 607-612, 1988.

[24] J. T. Zhang, L. S. Liu, P. C. Zhai, Q. J. Zhang. "Experimental and numerical researches of dynamic failure of a high strength alumina/boride ceramic composite," High-Performance Ceramics V, Pts 1 and 2, vol. 368-372, pp. 713-716, 2008. 